\section{Zum Zerfallsschema des J128}

Von Th. St rib e l

Hochspannungslaboratorium Hechingen,

Abt. des Max-Planck-Instituts für Physik der Stratosphäre

(Z. Naturforschg. 10 a, 797 [1955] ; eingegangen am 29. Juli 1955)

Es ist gelegentlich einer Arbeit, in der der Übergang $\mathrm{J}^{128} \rightarrow \mathrm{Te}^{128}$ mit einem Verzweigungsverhältnis $\mathrm{K}+\beta^{+} / \beta^{-}$von etwa $5,3 \%$ massenspektrometrisch festgestellt wurde, von Reynolds${ }^{1}$ darauf hingewiesen worden, da $\beta$ das von $\mathrm{S}$ i e $\mathrm{g} \mathrm{b}$ a h $\mathrm{n}$ und $\mathrm{H}_{\text {o }} \mathrm{e}^{2}$ vorgeschlagene Zerfallsschema des $\mathrm{J}^{128}$ nicht als gesichert gelten kann, solange nicht nachgewiesen ist, daß der von ihnen angenommene zweite $\beta^{-}$-Zweig von 1,59 MeV wirklich existiert. Tatsächlich wäre es ebenso denkbar gewesen, daß die beobachtete $428 \mathrm{keV}-\gamma$-Linie auf den

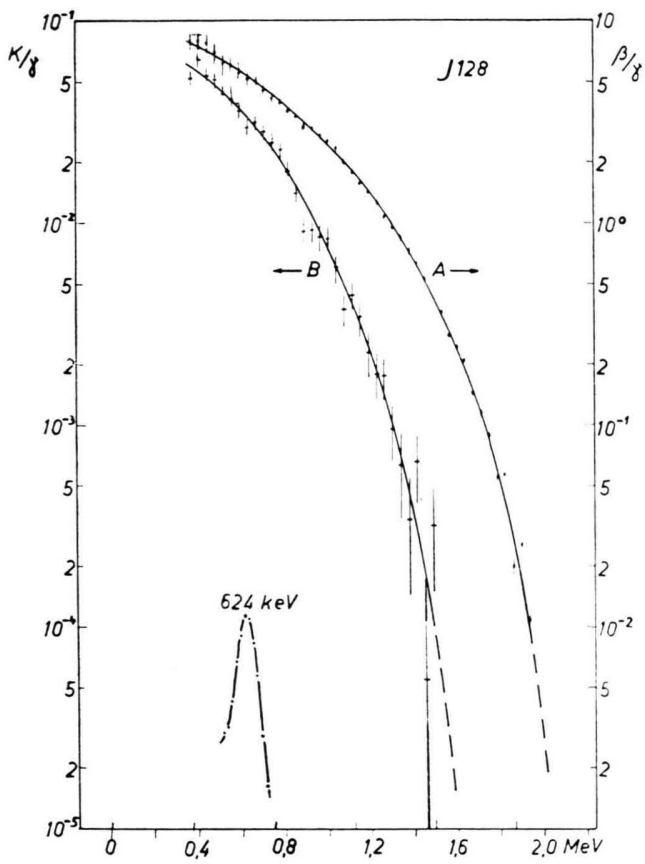

Abb. 1. Integrale Impulshöhenverteilung;

A: Gesamte $\beta$-Impulsverteilung. B: Verteilung der in Koinzidenz mit der $428 \mathrm{keV}-\gamma$-Linie auftretenden $\beta$-Impulse.

1 J. H. R e y n o ld s, Phys. Rev. 79, 789 [1950].

2 K. Si e g bahn u. N. Hol e, Phys. Rev. 70, 133 [1946].

3 W. C. B a r b e r, Phys. Rev. 72, 1157 [1947].
K-Einfang folgt und somit dem $\mathrm{Te}^{128}$ zuzuordnen wäre. (Für den Anteil des Positronenzerfalls des $\mathrm{J}^{128}$ konnte B a r b e ${ }^{3}$ eine obere Grenze von $0,2^{\%} \%$ der Zerfälle angeben; sehr wahrscheinlich ist er jedoch energetisch ganz verboten ${ }^{1}$.)

Mit einem $\beta$ - $\gamma$-Koinzidenz-Szintillationsspektrometer wurde das $\beta$-Spektrum des $\mathrm{J}^{128}$ untersucht. Als Detektoren dienen im $\gamma$-Kanal ein NaJ-Kristall, im $\beta$-Kanal ein $10 \mathrm{~mm}$ dicker „Sintilon“-Plastik-Luminophor von $22 \mathrm{~mm} \phi$. Abb. 1 zeigt die gemessenen integralen Impulshöhen-Verteilungen: Kurve A gibt die gesamte $\beta$-Impulsverteilung, Kurve $\mathbf{B}$ die der in Koinzidenz mit der $428 \mathrm{keV}-\gamma$-Linie auftretenden $\beta$-Impulse. Zufallskoinzidenzen sind bereits abgezogen, der zeitliche Abfall wurde durch Normierung auf die $\gamma$-Impulszahl berücksichtigt. Die zur $\beta$-Energieeichung benutzte innere Konversionslinie des $\mathrm{Ba}^{137 \mathrm{~m}}(624 \mathrm{keV})$ ist ebenfalls eingezeichnet.

Das Auftreten einer maximalen $\beta$-Energie von etwa 1600 $\mathrm{keV}$ in Koinzidenz mit der $428 \mathrm{keV}-\gamma$-Linie bestätigt das von Siegbahn und Hole vorgeschlagene Zerfallsschema. Da weitere $\gamma$-Übergänge außer der $428 \mathrm{keV}$-Linie nicht vorhanden sind, muß demnach der K-Einfang zum Grundzustand des $\mathrm{Te}^{128}$ führen. Dieser Übergang ist sehr wahrscheinlich erlaubt ; denn aus der einem verbotenen Übergang entsprechenden Energie des K-Einfanges würde nach den Tafeln von $\mathrm{F}$ e e $\mathrm{n}$ b e r g und T ri g g ${ }^{4}$ ein wesentlich kleineres $\mathrm{K} / \beta^{+}$-Verhältnis folgen, als es der von $\mathrm{B}$ a r b e $\mathrm{r}^{3}$ angegebenen oberen Grenze für den Positronenzerfall entspricht. Andererseits läßt sich auch der 2,02 MeV- $\beta^{-}$-Zweig mit einem $\log f t$-Wert von 5,9 noch als erlaubt einordnen, so daß dem Ausgangskern $\mathrm{J}^{128}$ mit großer Wahrscheinlichkeit Gesamtdrehimpuls und Parität $1+$ zuzuschreiben sind. Diese Zuordnung ist auch nach dem Schalenmodell ${ }^{5}$ zu erwarten, nach dem den Nukleonenzahlen $53-75$ die Termkombinationen $d_{5 / 2}-d_{3 / 2}$ oder $\mathrm{d}_{5 / 2}-\mathrm{g}_{7 / 2}$ entsprechen, die beide Kernspin 1 mit gerader Parität ergeben.

Dem $\beta$-Übergang zum $428 \mathrm{keV}$-Niveau des $\mathrm{Xe}^{128}$ entspricht ein $\log f t$-Wert von 6,7 , der ihn sicher in die Klasse $\Delta I=0,1$ mit Paritätsänderung einreihen läßt. Das $428 \mathrm{keV}$-Niveau hat demnach ungerade Parität und den Gesamtdrehimpuls 1 oder 2 ; eine Entscheidung zwischen diesen Werten kann nur eine Messung des Konversionskoeffizienten liefern, die bis jetzt wegen der Kleinheit desselben nicht möglich gewesen ist.

Herrn Prof. Dr. E. S c h o p p e r danke ich für sein Interesse an dieser Untersuchung, welche durch Mittel des Schwerpunktprogramms über die Deutsche Forschungsgemeinschaft in dankenswerter Weise unterstützt wurde.

${ }^{4}$ E. F e e n berg u. G. Trigg, Rev. Mod. Phys. 22, 399 [1950].

5 M. G. Mayer, S. A. Moszkowski u. L. W. Nordh e i m, Rev. Mod. Phys. 23, 315 [1951].

\section{Zur $\gamma$-Strahlung des Tellur-129}

Von Th. Stribel

Hochspannungslaboratorium Hechingen, Abt. des Max-Planck-Instituts für Physik der Stratosphäre (Z. Naturforschg. 10 a, 797-798 [1955]; eingeg. am 8. September 1955)

Über die beim $\beta$-Zerfall des 72 min-Te ${ }^{129}$ auftretenden $\gamma$-Ủbergänge lagen bis vor kurzem nur Absorptionsmessungen vor ${ }^{1}$, die Energien von 0,3 und $0,8 \mathrm{MeV}$ ergeben hatten.
Mit einem Szintillations- $\gamma$-Spektrometer wurden an diesem Isotop neue Messungen vorgenommen. In ungefährer Übereinstimmung mit neuerdings von $\mathrm{M}$ a $1 \mathrm{lmann}$ u. a. ${ }^{2}$ veröffentlichten Ergebnissen finden wir eine starke $\gamma$-Linie von $(460 \pm 10) \mathrm{keV}$ und eine Linie von etwa 10-mal geringerer

1 L. E. G l e n d e n i n, National Nuclear Energy SeriesPlutonium Project Report 9, 979 [1951].

${ }^{2}$ C. A. M a $11 \mathrm{~m}$ a $n \mathrm{n}$, A. H. W. A t e n jr., D. R. B e s u. Clara M. de M c Mill a n, Phys. Rev. 99, 7 [1955]. 